Instituto Internacional de Investigación y Desarrollo Tecnológico Educativo INDTEC, C.A.

DOI: https://doi.org/10.29394/scientific.issn.2542-2987.2017.2.5.18.341-355

OAl-PMH: http://www.indteca.com/ojs/index.php/Revista Scientific/oai

\title{
La Interdisciplinariedad y la Hermenéutica como Recursos Pedagógicos para la Elaboración de Proyectos de Aprendizaje
}

\author{
Autora: Lizbeth Teresa Villamizar Vega \\ Complejo Educativo "Dr. Raúl Quero Silva", IUTAJS-IUPSM \\ villamizarlizbeth1503@gmail.com \\ Mérida, Venezuela
}

\section{Resumen}

Los llamados Proyectos de Aprendizaje (P.A.), se consideran una estrategia metodológica concebida en la escuela y para la escuela, que deben elaborarse en un año escolar producto de un trabajo colaborativo entre la comunidad educativa: estudiantes, docentes y directivos, pero donde algunos docentes no tienen idea clara de la vinculación que pueden darle a los contenidos programáticos de las asignaturas que imparten con el proyecto de aprendizaje, asimismo desconocen que la interdisciplinariedad y la hermenéutica son recursos pedagógicos que le permitirán tanto a docentes como alumnos la búsqueda de la respuesta a ¿qué significa su accionar diario?, es aquí donde se reconoce la necesidad de la visión holística de los docentes que administran las distintas unidades curriculares en las escuelas técnicas, donde deben buscar a través de los P.A. que los alumnos demuestren su capacidad de otorgarle a los nuevos conocimientos, funcionabilidad instrumental y utilidad para resolución de problemas presentes y futuros, que puedan crear seres humanos críticos, reflexivos, humanistas, liberadores y ambientalistas, que beneficien a la comunidad donde se encuentran inmersos, donde la solución del problema sea resuelto con la interpretación de los conocimientos transmitidos, así como con las nuevas competencias interdisciplinarias para buscar soluciones reales a problemas reales. aprendizaje.

Palabras clave: interdisciplinariedad; hermenéutica; proyectos de

Fecha de Recepción: 07-04-2017

Fecha de Aceptación: 05-05-2017 


\title{
Interdisciplinarity and Hermeneutics as Pedagogical Resources for the Elaboration of Learning Projects
}

\begin{abstract}
The so-called Learning Projects (AP) are considered a methodological strategy conceived in the school and for the school, that must be elaborated in a school year product of a collaborative work between the educative community: students, teachers and managers, but where some teachers Have no clear idea of the linkage they can give to the syllabus contents of the subjects they teach with the learning project, they also do not know that interdisciplinarity and hermeneutics are pedagogical resources that will enable both teachers and students to seek the answer to What does their daily action mean? It is here that the need for a holistic view of the teachers who manage the different curricular units in the technical schools is recognized, where they must search through the APs That the students demonstrate their ability to grant new knowledge, instrumental functionality and usefulness to solve present and future problems that can create critical, reflective, humanistic, liberating and environmental human beings that benefit the community where they are immersed, Where the solution of the problem is solved with the interpretation of the transmitted knowledge, as well as with the new interdisciplinary competences to look for real solutions to real problems.
\end{abstract}

Keywords: interdisciplinarity; hermeneutics; learning projects.

Date Received: 08-04-2017

Date Acceptance: 05-05-2017 


\section{Introducción}

El derecho a la educación puede definirse como la capacidad (innata) al ser humano de recibir (enseñanza) formal, así como, de exigir del Estado la garantía de brindársela, ya que es su deber u obligación estatal, esta función inherente al Estado tiene como fin el desarrollar en el individuo (niño, niña y adolescente) pensamientos críticos, reflexivos, solidarios y conscientes, que le permitan contribuir en su pleno desarrollo y discernimiento, así como a la transformación social integral y sustentable del país.

Este planteamiento está fundamentado en el artículo 102 de la Carta Magna venezolana, que permite tanto al docente venezolano como al alumno, aprovecharse de técnicas y estrategias adecuadas para que puedan ser aplicadas y abordadas en diversas situaciones reales y que alcancen así, los propósitos formativos y educativos para que, tanto los alumnos como los docentes puedan aprovechar las habilidades y aptitudes, las competencias, destrezas y las estrategias que le permitan construir, una conciencia que implique el reconocimiento de la importancia, tanto del trabajo individual como colaborativo con lo que se estaría formando, un ciudadano integral u holístico.

\section{Diagnóstico Situacional Educativo Venezolano}

El Sistema Educativo Bolivariano (SEB) estructura sus niveles de la siguiente manera: Educación Inicial Bolivariana (niveles Maternal y Preescolar); Educación Primaria Bolivariana (de 1ํa 6음ado); Educación Secundaria Bolivariana, en sus dos alternativas de estudio (Liceo Bolivariano, de $1^{\circ}$ a $5^{\circ}$ año; y Escuela Técnica Robinsoniana y Zamorana, de $1^{\circ}$ a $6^{\circ}$ año); Educación Especial; Educación Intercultural y Educación de Jóvenes, Adultos y Adultas (incluye la Misión Robinson 1 y 2 y la Misión Ribas). La Escuela Técnica Robinsoniana y Zamorana tiene como finalidad ofrecer una formación dirigida al desarrollo endógeno, fomentando habilidades, destrezas, valores y 
virtudes que fortalezcan esta orientación; al mismo tiempo que propicia el pensamiento crítico, reflexivo, humanista, liberador y ambientalista.

También, proporciona herramientas para la investigación, que permitan el desarrollo de proyectos productivos, sociales y culturales que beneficien a la comunidad, logrando una formación integral, sustentada en un compromiso social para la transformación del país. Asimismo, garantiza la formación especializada a las y los adolescentes y jóvenes en los campos científico, humanístico, artístico, técnico y tecnológico, para su futura incorporación al proceso productivo social y la prosecución a la Educación Superior.

La Ley Orgánica de Educación publicada en Gaceta Oficial No. G.O. 5929 Extraordinaria, de fecha sábado 15 de agosto del 2009, fundamenta este principio constitucional en el artículo 15 que reza de la siguiente manera: La educación, conforme a los principios y valores de la Constitución de la República y de la presente Ley, tiene como fines: 1. Desarrollar el potencial creativo de cada ser humano para el pleno ejercicio de su personalidad y ciudadanía, en una sociedad democrática basada en la valoración ética y social del trabajo liberador y en la participación activa, consciente, protagónica, responsable y solidaria, comprometida con los procesos de transformación social y consustanciada con los principios de soberanía y autodeterminación de los pueblos, con los valores de la identidad local, regional, nacional con una visión indígena, afrodescendiente, latinoamericana, caribeña y universal.

El sistema educativo venezolano, de la misma manera orienta la formación de los ciudadanos y ciudadanas con principios de identidad local, regional y nacional, además de crear seres humanos que puedan, desde su conciencia ciudadana, favorecer su entorno, por lo que el artículo 27 de la Carta Magna, señala que los alumnos deben participar en una actividad que beneficie al respectivo plantel o a la comunidad en la que se encuentran inmersos y que redundaría en su formación integral, dicho requerimiento va 
aunado a los demás requisitos legales establecidos para la culminación y obtención del título de bachiller o medio técnico.

\subsection{Los Proyectos de Aprendizaje}

Es aquí, donde para dar cumplimiento a esta norma jurídica, se estableció que deben planificarse y elaborarse los Proyectos de Aprendizaje (P.A.), los cuales según Amarista y De Navarro (2001) citados por Castillo y Jiménez (2013)

son una estrategia metodológica concebida en la escuela, para la escuela y los educandos; elaborada por el conjunto de actores de la acción_escolar, incluida la participación de la familia_y otros integrantes de la comunidad. Implica acciones precisas en la búsqueda de solución a los problemas de tipo pedagógico; ejecutadas a corto, mediano o largo plazo, en atención a las particularidades de cada proyecto que se desarrolle en las distintas etapas o grados de estudio. (pág. 32).

Los P.A. son un instrumento para planificar la formación educativa, mediante el cual el docente puede organizar y programar el proceso de la adquisición de nuevos conocimientos durante el año escolar respectivo, con miras a la formación de ciudadanos y ciudadanas con visión global de la realidad que los rodea. Dichos P.A. deben facilitar y mejorar la relación existente entre distintas unidades curriculares además de los contenidos programáticos establecidos por las normas jurídicas que rigen el sistema educativo, en este, el sistema educativo venezolano.

De la misma manera, dichos proyectos reconocen que con una misma actividad, pueden ser vinculados diversos aspectos no solo pedagógicos, comunitarios sino también familiares, porque con dichos proyectos de aprendizaje en el ser integro que quiere formarse se sembrarán principios y valores morales y éticos que a su vez le permitirán irse adaptando en cada etapa del sistema educativo, y en cada área de aprendizaje desarrollará 
habilidades y destrezas en función de sus propias características y sus propias necesidades.

Un Proyecto de Aprendizaje (P.A.) proporciona herramientas para que el alumno construya el conocimiento enfatizando en el proceso de investigación como componente que estimula no solo la actividad de recolectar y organizar datos per sé, sino el acto de conocer y de producir aprendizajes significativos. Formulando un problema de investigación que tome en cuenta la experiencia de los alumnos en su entorno escolar, familiar y comunitario, es que los aprendices entenderán que se convierten en un ser holístico o integral, puesto que cada área de conocimiento se vincula con un novedoso tema $u$ objetivo de estudio o de investigación, lo que evitaría al mismo tiempo, una fragmentación o parcelamiento del conocimiento, puesto que se enmarca la concepción de la ejecución del Proyecto de Aprendizaje en la interdisciplinariedad.

Para el desarrollo de todo P.A. son los estudiantes los que al inicio de cada año escolar seleccionan un tema o problema a investigar, aunque en dicha selección suelen encontrarse con serias dificultades. En las escuelas técnicas sobre todo, son los alumnos los que seleccionarán el tema, y este deberá estar enmarcado en las unidades curriculares del área de aprendizaje; hay instituciones educativas técnicas que imparten su educación en áreas como Servicios Administrativos, Aduana, Informática, Deporte, y para los jóvenes son unidades curriculares ajenas desde la perspectiva de la educación en ciencias; por otro lado, dicho proyecto debe estar vinculado con el Proyecto Educativo Integral Comunitario (PEIC) de la institución y por último, se debe destacar con mucha consternación, que un número significativo de docentes, no han planificado desde el inicio del año escolar las herramientas adecuadas para la asesoría en la escogencia del tema, puesto que ellos mismos no reconocen los problemas que les circundan y que están contenidos en el PEIC. 
De acuerdo a Rojas (2016),

Actualmente, al profesional de la docencia se le cuestionan viejas concepciones de enseñanzas, que, en cierta medida, no toma en cuenta los valores sociales del aprendizaje, en tanto su praxis muchas veces se limita a transmitir conocimientos, sin considerar la participación y autonomía del alumno, así como también la creatividad y la innovación en el acto pedagógico. (pág. 252).

En base a ello, existen docentes que no vinculan sus contenidos programáticos con el proyecto de aprendizaje, ni por separado ni en conjunto con las otras áreas del conocimiento, puesto que al inicio del año escolar, no se concretan reuniones multidisciplinarias para que sean ellos quienes les aporten a los alumnos, las ideas más acordes a los P.A., en el entendido que los docentes que imparten unidades curriculares con contenido programático similar, puedan planificar actividades unificadas para ser evaluadas en una sola ocasión, por lo que terminan asignándose e incluso evaluándose contenidos semejantes, hasta en tres oportunidades.

El proyecto de aprendizaje, lapso por lapso, debe irse complementando en función de cumplir con una estructura formal de un trabajo escrito con pautas internas adaptadas a las normas APA vigentes y expuesto al finalizar cada lapso, además de exigir una correlación de cada unidad curricular, y aun en esta fase los estudiantes, se siguen encontrando con dificultades, puesto que, se escapan de su control los factores internos como el desconocimiento del contexto de la interdisciplinariedad por parte de los docentes, ya que ellos no tienen una visión global exigida para el acompañamiento del estudiantado.

\subsection{La Interdisciplinariedad y la Hermenéutica}

No se trata pues, que con la interdisciplinariedad se puedan reducir unas disciplinas a otras, sino relacionarlas para enriquecer las aportaciones propias. 
El Consejo de Educación Secundaria de Uruguay (2016) señala que,

La interdisciplinariedad implica la receptividad de crítica y autocrítica en todas direcciones y se transforma en un proceso gradual de enriquecimiento curricular. Este tipo de trabajo requiere, por parte de sus participantes, una actitud de permeabilidad y apertura hacia las otras disciplinas y el quebrantamiento de estructuras rígidas individuales. (pág. 6).

Asimismo, Rivera (2015) sostiene

En este sentido, es importante acotar que no es la suma de disciplinas ni la idea de una división de trabajos que encuentran puntos en común (búsqueda de interfaces) la que dará vida a investigaciones interdisciplinarias, sino la introducción de la complejidad al mundo de las Ciencias Sociales como conciencia del todo, es decir, focalizar la organización de las disciplinas acostumbradas a optimizar las diferencias de sus subsistemas de pensamiento en paradigmas conservadores. (pág. 14).

En resumen, una de las condiciones importantes para hacer un planteamiento desde la interdisciplinariedad, es el hecho de que en la solución de un problema cada especialista de un área o disciplina comprenda el punto de vista, los problemas y las dificultades de los demás y éstos a su vez, hagan lo mismo.

Aplicando la interdisciplinariedad como recurso pedagógico para el diseño de estrategias en la construcción de los proyectos de aprendizaje, se entenderá la necesidad de egresar jóvenes que se destaquen por el sentido de reconocer el SER, HACER Y CONVIVIR, pilares fundamentales del sistema educativo venezolano, respetando así, lo estipulado en la normativa jurídica del Curriculum Bolivariano, con este fin se favorece y se espera promover la formación integral de los Mediadores Educativos (Personal Directivo, Docentes) y darle sentido de pertenencia e interacción con el entorno. Es aquí donde se reconoce la necesidad de la visión holística delo los docentes que administran las distintas unidades curriculares en las escuelas técnicas. 
De acuerdo a Alarcón (2013),

Los maestros como responsables de la formulación de proyectos deben estar capacitados, no solo cono conocimientos referidos a las características evolutivas de los niños en edad escolar, al diseño y administración de estrategias metodológicas y de evaluación que generen y garanticen aprendizajes significativos, la selección de técnicas y recursos para el aprendizaje que potencien la construcción de conocimientos, sino que además deberá tener competencias que le permitan formular, ejecutar y evaluar Proyectos de Aprendizajes creativos, innovadores, integradores, contextualizados, que propicien la participación de los actores del hecho educativo, capaces de generar un pensamiento crítico y reflexivo en los estudiantes, así como la construcción de conocimientos a partir de la investigación como eje transversal del proceso educativo y que den respuesta a las necesidades de la localidad. (pág. 25).

El docente en la elaboración de estos P.A. debe aprovecharse de diversas estrategias y herramientas, entre ellas la hermenéutica, vista ésta como una metodología con la que se pueden articular los contenidos programáticos con los valores, conocimientos predefinidos del alumnado, así como llamar y mantener la atención de los sujetos de la interpretación y resolución de la realidad social. Por ende, en el proceso de enseñanza y aprendizaje el docente no puede aspirar a una simple repetición de conceptos, sino fomentar la vinculación de la nueva información introducida en el acto didáctico con las experiencias previas.

Puede considerarse la hermenéutica como un método alternativo en el abordaje de la educación que se fundamente en la participación intrínseca del hombre en la búsqueda de la respuesta a qué significa su accionar diario, por qué es parte del mundo y de cómo puede interpretar todo lo que sucede, qué le permite relacionarse con otros sujetos y comunicarse con sus pares.

En este sentido la hermenéutica es una metodología que permite vincular la interpretación del individuo sobre la realidad circundante y en 
procura del significado de la acción humana para poder abordar los problemas de la sociedad que, desde su esencia, le permite aprehender y comprender las necesidades sociales y la búsqueda de la solución.

Desde esta perspectiva, no se podría hablar propiamente de una metodología, sino de una forma de estar en el mundo y de cómo a través de la experiencia se interpreta lo que pasa, las interacciones con los otros sujetos y si se quiere, los discursos que a través del diálogo los actores se comparten. La hermenéutica, por tanto, debe servir para generar un acercamiento a textos, leyes, así como autores y doctrinarios que permitan comprender aquello que ellos pretenden decir.

La teoría constructivista es una de las teorías que utilizan la hermenéutica como herramienta en la educación, formación y transformación, ésta se orienta a interpretar los fenómenos sociales, y, por consiguiente, los educativos y se interesa por el estudio de los significados e intenciones de las accionas humanas, desde la perspectiva de los propios agentes sociales. Asimismo, consideran que el ser humano es un espectador imparcial de los fenómenos, cualquier conocimiento de las cosas viene mediado por una serie de prejuicios y expectativas que orientan y limitan nuestra comprensión.

La actividad hermenéutica hace posible el proceso de adquisición de saberes y de interpretación, por lo que constituye el soporte y fundamento de una personalidad crítica, reflexiva, interpretativa que, en consecuencia, otorga al individuo la capacidad de percibir "literalmente" la realidad para poder implementar los cambios necesarios y oportunos a favor del bienestar individual y colectivo. Para Martínez y Ríos (2006) la hermenéutica pretende recuperar el juicio reflexivo como forma de conocer.

La tendencia a que los docentes interpreten hermenéuticamente la realidad y los textos, para la selección de los problemas que abordarían a través de los proyectos de aprendizaje, en los distintos niveles del sistema educativo venezolano, específicamente en el nivel medio técnico, los apartaría 
de conductas indiferentes, los ubicaría en el aquí (aulas, familia, comunidad educativa) y el ahora humanistas, intérpretes, de la realidad e ideas de quienes los rodean. En tal sentido Rojas (2016), refiere

Por lo tanto, se requiere un profesional de la docencia capaz de identificar problemas, investigarlos desde una nueva lógica de interrogación y presentar soluciones a los mismos; también se requiere realizar acciones necesarias para que las soluciones sean posibles; $y$ ante estas acciones el profesional de la docencia en educación, juega un papel decisivo frente a los nuevos retos que se presentan, tanto en las aulas de clases como en el contexto de la vida cotidiana. Es por ello, que una de las demandas básicas de la sociedad, es la de contar con profesionales capacitados y eficientemente preparados para enfrentar el desafío de educar desde la unidad y la diversidad, pero también desde la certeza y la incertidumbre. (pág. 255).

También se propicia la inserción del ser humano en la época que le toca vivir, dada su capacidad para discernir y por ser portador de la palabra, lo que le da la posibilidad de visualizar el lugar que ocupa dentro de un determinado paradigma sociocultural para que una vez comprendido y repensado, sea capaz de reinterpretar el discurso.

Por ello lo que deben buscar los docentes a través de los P.A. es que los alumnos demuestren su capacidad de otorgarle a los nuevos conocimientos, funcionabilidad instrumental y utilidad para resolución de problemas presentes y futuros. Con ello se podrá decir que el docente no solo trasmite un conocimiento, sino que también forma competencias para buscar soluciones. En el proceso de enseñanza no se debe individualizar o parcializar el conocimiento, por ello la interdisciplinariedad, reconoce la vinculación entre áreas de distinto conocimiento que pueden trabajar de la mano, esto permitirá al alumno tener independencia y su propio ritmo, se promueve el trabajo colaborativo y con ello, aprenden habilidades sociales. 
De acuerdo al Consejo de Educación Secundaria de Uruguay (2016),

Sin duda alguna, estas condicionantes implican algunos desafíos concretos para el trabajo en el aula y en la coordinación que deben ser considerados. Una de las consideraciones centrales en el trabajo interdisciplinario de las duplas y tríos será identificar oportunidades de ensenar el pensamiento disciplinar específico, mientras se realizan abordajes interdisciplinarios de los tópicos o problemas seleccionados en la planificación. Probablemente resulte trabajoso encontrar este punto de equilibrio, pero una vez acordados los caminos, la enseñanza y el aprendizaje se aproximarán a abordajes más complejos, ricos y cercanos a las realidades que rodean a los alumnos. (pág. 7).

\section{Conclusión}

En síntesis, la sociedad requiere que los conocimientos sean aprendidos y aprehendidos, pero a través de la implementación de nuevos métodos, como: el P.A., la hermenéutica y la interdisciplinariedad, que permitan superar la inercia mental y crear un clima en el aula que dé libertad de pensamiento, libertad mental, que estimule la participación cooperativa o grupal, en la conformación de conceptos propios que les permitan a los alumnos afrontar con sensibilidad los cambios en el tiempo. Estrategias que pueden proporcionar al docente la obtención, no solo de buenas preguntas, sino que sabe hacer buenas preguntas con las cuales, al construir los significados propios que puedan aportar soluciones a la realidad, ayudándola a transformarla.

En conclusión, el docente debe manifestar apertura al cambio, ser facilitador de ambientes de aprendizaje y un mediador que genere en el alumno, reflexión y análisis de la realidad que lo rodea, que le conozca su entorno familiar y haga alianza con él para poder ayudarlo, tener competencias en el manejo de conflictos, es decir, constituirse en un factor de cambio social. En el caso particular de los docentes de las escuelas técnicas, deben centrarse en una metodología de la interpretación, y en el aprender haciendo. 
Interpretar y analizar la realidad no es para nada fácil, por lo que el docente debe brindar las técnicas y estrategias adecuadas para que los alumnos aborden la temática y se alcancen los propósitos iniciales y así, se puedan aprovechar las competencias, destrezas, habilidades y aptitudes que gracias, al diagnóstico inicial, todo docente puede determinar, dejar participar al alumno para que construya su conocimiento con el trabajo individual y colaborativo con lo que se estaría formando un ciudadano integral u holístico, fundamentado en el artículo 102 de la Carta Magna venezolana que reconoce entre otros, el derecho a la educación como derecho humano y como deber u obligación del Estado, función inherente a la finalidad gubernamental de desarrollar pensamientos críticos, reflexivos, solidarios, conscientes en el niño y el adolescente para que contribuyan a la transformación social integral y sustentable.

\section{Referencias}

Alarcón de C. (2013). Proyecto de Aprendizaje: Estrategia Metodológica para su formulación. Trabajo de Ascenso como requisito parcial para optar a la Categoría de Profesor Agregado, Universidad Nacional Experimental de Guayana, Venezuela.

Asamblea Nacional de la República Bolivariana de Venezuela (1999). Constitución de la República de Venezuela. G.O. 36860, de fecha 30 de diciembre de 1999.

Asamblea Nacional de la República Bolivariana de Venezuela (2009). Ley orgánica de educación. Gaceta Oficial No. G.O. 5929E. De fecha 13 de agosto.

Castillo, E. y Jiménez, F. (2013). Los Proyectos de Aula y el Desarrollo de Destrezas con Criterios de Desempeño en el Área de Ciencias Naturales con los/las Estudiantes del Cuarto Año de Educación General Básica de la Unidad Educativa "10 De Enero del Cantón De 
San Miguel”, Provincia Bolívar, Año Lectivo 2013-2014. Trabajo de Tesis presentado en opción a obtener el Título de Licenciados en Ciencia de la Educación, Mención: Educación Básica, Universidad Estatal de Bolívar, Ecuador.

Consejo de Educación Secundaria de Uruguay (2016). Interdisciplinariedad y Coenseñanza. Aportes para la reflexión e implementación en aulas de enseñanza media. Recuperado de:

https://www.ces.edu.uy/index.php/component/phocadownload/categor y/31-propuestas-de-didactica?download=159:interdisiplinariedad-ycoensenanza

Martínez, A. y Ríos, F. (2006). Los conceptos de conocimiento, epistemología y paradigma, como base diferencial en la orientación metodológica del trabajo de grado. Revista electrónica de epistemología en Ciencias Sociales, Nro. 25, ISSN: 0717-554X. [Artículo en Línea]. Recuperado de:

http://www.cintademoebio.uchile.cl/index.php/CDM/article/viewFile/259 $\underline{60 / 27273}$

Rivera, R. (2015). La Interdisciplinariedad en las Ciencias Sociales. Revista Reflexiones, Revista Facultad de Ciencias Sociales de Universidad de Costa Rica, Volumen 4, Numero 1. [Artículo en Línea]. Recuperado de:

http://revistas.ucr.ac.cr/index.php/reflexiones/article/view/20871/21013 Rojas, N. (2016). Formación del Docente y Pedagogía de la Complejidad en el Contexto de la Educación. Revista Scientific, 1(1), 249-265. Recuperado de:

https://doi.org/10.29394/scientific.issn.2542-2987.2016.1.1.14.249-265 


\section{Lizbeth Teresa Villamizar Vega}

e-mail: villamizarlizbeth1503@gmail.com

Nacida en Venezuela. Politólogo, Abogado, Especialista en Derecho Tributario, MSc. en Cs. Políticas, Cursante del Programa de Profesionalización de la ULA Licdo. en Educación, Doctorando en Ciencias de la Educación en la Universidad Fermín Toro, Docente Universitaria (Pre y Postgrado) en instituciones Públicas y Privadas, Tutora, Asesora y Jurado en T.E.G. (Pre y Postgrado) en instituciones Públicas y Privadas. Complejo Educativo "Dr. Raúl Quero" Extensión Mérida (IUTAJS-IUPSM), Docente en Educación Media y Técnica General U.E. "Colegio Inmaculada Concepción" Mérida. 